\title{
Tobacco Treatment Specialists in France
}

\author{
Ivan Berlin, Jean Perriot, François Letourmy on behalf of the French Society for Tobaccology
}

John R. Hughes lists strong arguments to implement a new profession: tobacco treatment specialists (TTS) to cover clinical activities as opposed to public health activities in helping smokers quit (Hughes, 2007). To our knowledge France is the only country that has introduced as early as 1985 an evidence-based postgraduate course to train TTS called 'tobaccologist'. The first interuniversity teaching course was launched for the university year 1985-1986 at the University Paris XIMedical School. Later, several medical schools have associated by region to organise postgraduate training courses for physicians who took the decision to be trained for smoking cessation. As of today, France has five interuniversity diploma courses to train tobaccologists: West (six medical schools), North-East (three medical schools), East (five medical schools), South (four medical schools) and Paris (two medical schools).

These trainings comprise 9 to 15 full-day teaching courses in several sessions over one school year. The programs are roughly similar across regions and include almost all aspects of tobacco, such as: epidemiology of smoking and smoking-related health risks, nicotine's pharmacology, genetics of tobacco dependence. Particular attention is paid to tobacco use in specific populations like pregnant women, adolescents, psychiatric populations and persons with other addictive disorders. Courses also include assessments of clinical features: tobacco/nicotine dependence, withdrawal signs and symptoms and pharmacological and nonpharmacological treatments with demonstrated efficacy for smoking cessation. The evidence-based nature of knowledge is taught in methodological courses specific for tobacco-related issues.

In all five interuniversity training courses attendees should participate in a 5-day training at a senior tabaccologist's office and write up a thesis on a tobacco-related topic. The attendees must pass a written and oral exam. The final note is the sum of the thesis, oral and written exam. A total of 1,798 students obtained their tobaccologist diploma between 2000 and 2007 (Table 1).

Although this is not a recognised discipline by the Order of Physicians, those physicians who have completed these courses can use, with the agreement of the Order of Physicians, the label 'tobaccologist'. Increasingly, more midwives and some nurses attend these courses and the Order of Midwives now allows the use of the title 'midwife tobaccologist'.

Those who obtained the diploma of tobaccologist may qualify to be a member of the French Society for Tobaccology, which allows encounters, participation in thematically oriented working groups and in the annual congress of the society.

More than 200 tobaccologists are trained per year by the five postgraduate courses (Table 1). State-funded positions are open specifically to tobaccologists in public hospitals all over the country, leading to a network of

\section{Table 1}

Number of Postgraduate Students Who Obtained Their Diploma of Tobaccologist (Tobacco Treatment Specialist) in France Between 2000 and 2007

\begin{tabular}{|c|c|c|c|c|c|c|c|c|c|}
\hline Years & 2000 & 2001 & 2002 & 2003 & 2004 & 2005 & 2006 & 2007 & Total \\
\hline Paris & 85 & 81 & 82 & 84 & 86 & 83 & 81 & 83 & 665 \\
\hline North-East & 31 & 35 & 29 & 35 & 32 & 31 & 30 & 33 & 256 \\
\hline West & & 28 & 32 & 37 & 34 & 28 & 27 & 25 & 211 \\
\hline East & 35 & 36 & 39 & 35 & 32 & 37 & 40 & 41 & 295 \\
\hline South & 23 & 38 & 46 & 47 & 46 & 48 & 58 & 65 & 371 \\
\hline Total & 174 & 218 & 228 & 238 & 230 & 227 & 236 & 247 & 1798 \\
\hline
\end{tabular}

Address for correspondence: Ivan Berlin, Service de Pharmacologie, Hôpital Pitié-Salpêtrière-Université P. \& Marie Curie, Faculté de médecineINSERM U677 47, bd de l'Hôpital, 75013 Paris. E-mail: ivan.berlin@psl.aphp.fr 
smoking cessation outpatient clinics. In 2008, there were 650 state-funded smoking cessation outpatient clinics with a mean of three tobaccologists per clinic for an estimated 10 million smokers, resulting in a 5000 smokers per tobaccologist ratio. This ratio is an underestimate because it does not take into account private practices. One hundred and sixty smoking cessation clinics are connected to a computerised network. This allows the use of a standardised electronic patient chart and data collection. The tobaccologists' activity is essentially clinical: to help smokers quit, but most of them have some preventive activities and participate as teachers in training sessions for the hospital personnel or in companies.

The existence of tobaccologists has changed the medical landscape. Self-referral by patients is no more the rule. Specialists of all kinds increasingly refer more smoking patients to the tobaccologists who provide extended care because a single quit attempt usually fails. Half of the patients are hard core smokers with psychiatric or addictive co-morbidities.

The universal healthcare system reimburses the visits at the tobaccologist's office at a similar level to that for a visit at a primary care physician. We aim to get a reimbursement at the level of the specialists but this will be a hard fight...

\section{Reference}

Hughes, J.R. (2007). Tobacco treatment specialists: A new profession. Journal of Smoking Cessation, 2, Suppl. 2-7. 